\title{
Development of Virtual Reference Station in Kinematic Schemes of Geodetic GPS Network Using the Method of Maximum Informative Zone
}

\author{
Arif Shafayat Mehdiyev, Ramiz Ahmed Eminov², Hikmat Hamid Asadov ${ }^{3}$ \\ ${ }^{1}$ National Academy of Aviation, Baku, Azerbaijan; ${ }^{2}$ State Oil Academy, Baku, Azerbaijan; ${ }^{3}$ Research Institute of Aerospace Infor- \\ matics, Baku, Azerbaijan. \\ Email: asadzade@rambler.ru
}

Received August $13^{\text {th }}, 2013$; revised September $13^{\text {th }}, 2013$; accepted September $20^{\text {th }}, 2013$

Copyright (C) 2013 Arif Shafayat Mehdiyev et al. This is an open access article distributed under the Creative Commons Attribution License, which permits unrestricted use, distribution, and reproduction in any medium, provided the original work is properly cited.

\begin{abstract}
The factual data on error of positioning in VRS GPS networks have been analyzed, where the mobile receiver is provided with VRS. The method of highly informative zone is suggested for removal of initial vagueness in selection of reference stations for purposes of development of VRS on the basis of minimum GPS network, composed of three reference stations. The recommendations on use of suggested method are given.
\end{abstract}

Keywords: GPS Receiver; Virtual Reference Station; Kinematic Schemes; Positioning; Geodetic Network

\section{Introduction}

It is well-known that at present time a networked RTK (NRTK) system, characterized with high accuracy makes it possible to carry out positioning upon distances between stations, equal to 70 - $100 \mathrm{~km}$ [1]. One of perspective ways for further increase of accuracy of RTK systems of positioning is a method of Virtual Reference Station (VRS), which is a model of reference station (RS), located at close proximity of MR [2]. Utilization of VRS makes it possible to carry out RTK positioning by accuracy of $2 \mathrm{~cm}$ in network of RS by distance between equal to $32 \mathrm{~km}$. According to work [3], major principles of development of VRS are as followings:

- Data from network of reference stations are transferred to processing center;

- Data of network is used for calculation of models of ionosphere, troposphere and orbital errors;

- The ambiguity of estimate of phase of carrier is fixed taking into account the base distances of network;

- The factual errors of base distances are determined with $\mathrm{cm}$ accuracy on the basis of measurements of fixed phase of the carrier;

- To forecast the total error of positioning of the user's mobile receiver the linear combination of errors' models are used;

- VRS is to be developed in close vicinity of mobile receiver.

Till now the question on well-grounded choice of place of VRS in RTK network and also the question on choosing of RS for positioning of user's MR are not solved in proper manner. Instead such heuristic criteria as "RS, which are closest to mobile receiver" and "closest placement of VRS to mobile receiver" are used. In this paper, we shall consider the possibility on substitution of heuristic criteria with information ones.

\section{Critical Review of Existing Methods}

According to work [4], in NRTK systems the distance between RS and mobile receiver is to be determined as a maximum value of radius of the circle which encompasses the zone, where signal of appropriate RS can be used efficiently together with sent signals having the format of International Radio technical Commission of Sea Service (RTCM) (Figure 1).

As it is noted in the work [5], in the system ROMPOS (Romania), which is the NRTK type system, the high accuracy of positioning is achieved as follows. Firstly, the MR sends its data relating its approximate position to the Control Center via Internet. The Control Center selects on the basis of received information the BS as a basic one which mostly closes to MR.

Then, the initial information, received from this RS is 


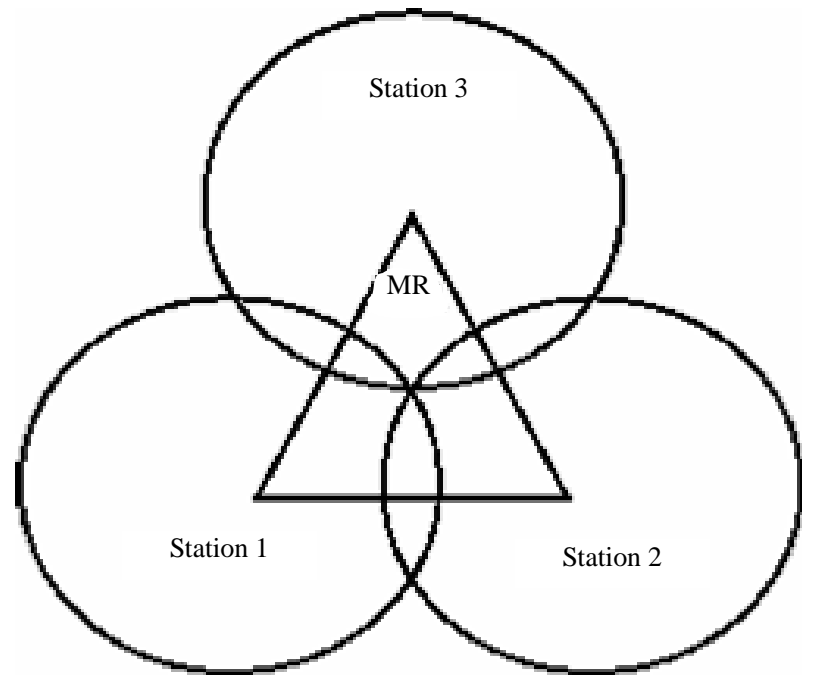

Figure 1. The ideal symmetrical configuration of placement of MR and reference stations [4].

to be corrected as a geometric position data taking into account the networks correction, which accords with approximate position of MP. At present time there are such competing technologies, as NRTK with VRS, NRTK with pseudo-reference station (PRS), method of parameters of correction on field, and also the concept of "master-apprentice" (MAC), and individualized correction of the same type (i-Max) [6].

According to work [7], in order to use a fast static or the real time kinematic (RTK) positioning with a centimeter accuracy at a large distances the reference networks were developed in some areas of Germany with step $30-50 \mathrm{~km}$. Within these networks the values of errors depending on distance were simulated, such as errors due to ionosphere and troposphere refractions and others, separately on satellites with high temporal resolution. Such a procedure leads to VRS concept, the output information of which is processed factually in the computer, using for this data of really existing RS.

According to the work [8], in order that the results of measurements of carrier's frequency's phase accepted in RS of network were transformed to the simulated result of phase measurements of VRS carrier's frequency's phase, some operations, including following, should be carried out:

1) Removal of ambiguities occurred due to double differencing of phase of carrier's frequency;

2) Calculation of models of errors, which depends on distances;

3) Development of ionosphere model, based on a linear ionosphere combination;

4) Development of geometrical model, containing the troposphere and orbital errors and based on linear combination of them.
In this case the processing of data can be carried out in central processor or can be distributed between the central processor and users. As it is noted in work [9], in the networked RTK systems of positioning the most critical component of positioning error is a differential ionospheres residual error between the network of RS and mobile receiver. According to the work [10], the concentration of electrons in ionosphere is suffered the heavy diurnal change. After the double differencing the troposphere error upon interference distances 60 - $80 \mathrm{~km}$ may surpass the ionosphere errors and reach the value of $2 \mathrm{~cm}$ during the 30 seconds time interval. At the same time, the question, related with choosing the place for development of VRS in the world practice is being solved till now in line with following heuristic considerations:

1) VRS should be located maximally close to MR of user;

2) For positioning of MR it is necessary to choose those RS which are located most closely to MR.

It is obvious that development of techniques and scientific technology require substitution of heuristic approaches by well-grounded scientific ones. Next we shall describe the proposed method for selection of place for development of VRS.

\section{Development of VSR Using the Method of Maximum Informative Zone Determination}

According to work [1], upon interpolation of VSR's error within the server's software the errors are to be interpreted on the basis of data of Reference Stations using the method of interpolation. At the same time the method of extrapolation also can be used (Figure 2).

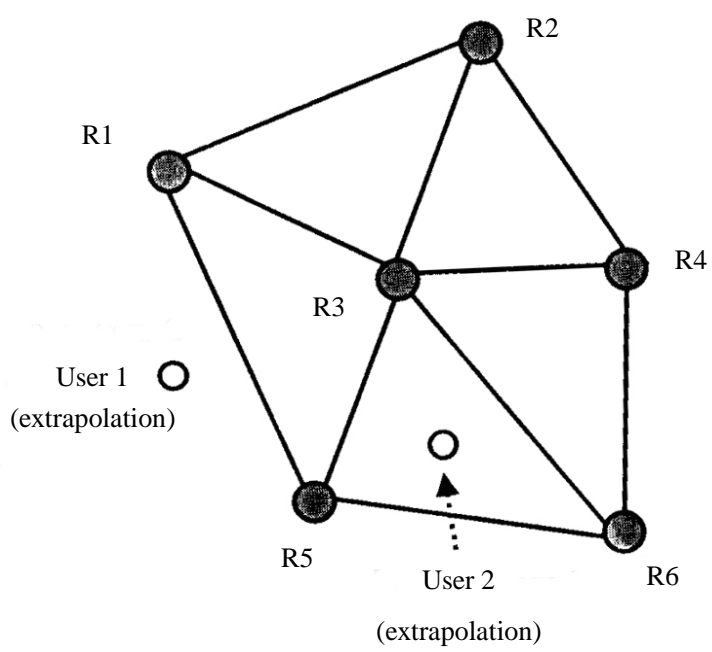

Figure 2. Illustration of cases where the carrying out of interpolation (user1) and extrapolation (user 2) are necessary. 
According to our opinion, the interpolation method for determining of MR-s position error in principle removes the possibility of carrying out of high-accuracy positioning due to existence of its inherent error componenterror of interpolation.

In view of abovementioned we suggest the information -interpolation method for determining the error of VRS positioning, the matter of which is following:

1) VRS should be located over MR, i.e. the places of their positioning coincide.

2) The interpolation of error is to be carried out and the interpolated assessment of the VRS (MR) position's error is to be geometrically determined.

3) The maximum informative zone in the area of location of VRS (MR) is to be determined, where the maximum amount of information can be received upon realization of some order of transfer of information from RS relating its position upon given limiting conditions;

4) For each position of VRS (MR), by purposes of interpolating such a triplet of RS should be chosen, the maximum informative zone of which determines the points of placement of VRS (MR).

The mathematical grounding of suggested method is as follows. Assume that there are six RS and the MR with unknown coordinates (Figure 3).

As a first step we choose stations RS1, RS3 and RS5. Taking into account the noisiness of the channel's signals due to effect of highly variable ionosphere error the techniques of multiple sending of data of own positions is used for transfer the information from RS to MR.

Thus, using the continual form of writing, the amount of information, received by VRS may be estimated as

$$
F_{1}=\int_{0}^{T_{\max }} M_{1} \mathrm{~d} t=\int_{0}^{T_{\max }} T \log _{2}[\psi(l)+1] \mathrm{d} T,
$$

where $T$-time of transfer of information from RS to

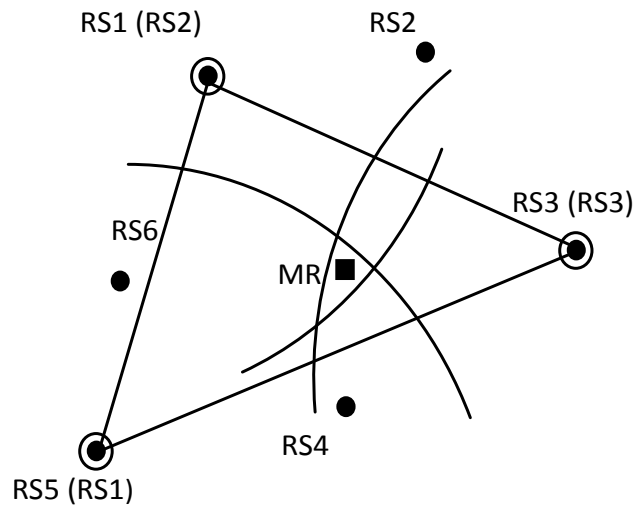

Figure 3. The graphical explanation of suggested method of highly informative zone; $\mathrm{MP}$-mobile receiver; RS1-RS6reference stations of initial network; (RS1)-(RS3)—reference stations used in triangular network.
VRS; $T_{\max }$-maximum value of $T ; \psi$-signal/noise ratio in transferred signal; $l$-distance between RS and VRS; $M_{1}=T \log _{2}[\psi(l)+1]$.

As a first approximation we have

$$
\psi(l)=\psi_{0}+\psi^{\prime} \cdot l,
$$

where $\psi_{0}$ - signal/noise ratio at reference stations; $\psi^{\prime}=\frac{\mathrm{d} \psi}{\mathrm{d} l}$.

Then with we introduce the function of relation between the distance $l$ and duration- $T$ :

$$
l=\varphi(T),
$$

Taking into consideration Formulas (1) - (3) we get

$$
F_{1}=\int_{0}^{T_{\max }} M_{1} \mathrm{~d} T=\int_{0}^{T_{\max }} T \log _{2}\left[\psi_{0}+\psi^{\prime} \cdot \varphi(T)+1\right] \mathrm{d} T .
$$

The limitation condition relating the total value of distances from MR to RS is formulated as follows

$$
F_{2}=\int_{0}^{T_{\max }} M_{2} \mathrm{~d} T=\int_{0}^{T_{\max }} \varphi(T) \mathrm{d} T=C_{1},
$$

where $C_{1}=$ const ; $M_{2}=\varphi(T)$.

It should be noted, that in case of monotonous type of function 3, the condition (5) may be interpreted as limitation for total duration of information reception.

In view of Equations (4) and (5) we can compose following equation of non-conditional variation optimization:

$$
\begin{aligned}
F_{3} & =\int_{0}^{T_{\max }} T \log _{2}\left[\psi_{0}+\psi^{\prime} \cdot \varphi(T)+1\right] \mathrm{d} T \\
& +\lambda \int_{0}^{T_{\max }} \varphi(T) \mathrm{d} T .
\end{aligned}
$$

where $\lambda$-Lagrange multiplier.

It is well-known, that the optimum function $\varphi_{\text {opt }}(T)$ should meet the Euler's condition

$$
\frac{\mathrm{d}\left(M_{1}+\lambda M_{2}\right)}{\mathrm{d} \varphi(T)}=0 .
$$

In view of Equations (6) and (7) we get

$$
\frac{T \cdot \psi^{\prime}}{(\ln 2)\left[\psi_{0}+\psi^{\prime} \cdot \varphi(T)+1\right]}+\lambda=0 .
$$

From formula (8) we derive

$$
\varphi(T)=-\left(\frac{\psi_{0}}{\psi^{\prime}}+\frac{1}{\psi^{\prime}}+\frac{T}{\lambda \cdot \ln 2}\right) .
$$

Taking into consideration the formulas (5) and (9) we get. 


$$
-\int_{0}^{T_{\max }}\left(\frac{\psi_{0}}{\psi^{\prime}}+\frac{1}{\psi^{\prime}}+\frac{T}{\lambda \cdot \ln 2}\right) \mathrm{d} T=C_{1}
$$

and

$$
\lambda=-\frac{T_{\max }}{2 \cdot \ln 2\left[\frac{\psi_{0}}{\psi^{\prime}}+\frac{1}{\psi^{\prime}}+\frac{C_{1}}{T_{m}}\right]} .
$$

Taking into consideration the formulas (8) and (11) we have

$$
l=\varphi(T)=\frac{2 T\left[\psi_{0}+1+C_{1} \psi^{\prime}\right]}{T_{\max } \cdot \psi^{\prime}}-\frac{\left(\psi_{0}+1\right)}{\psi^{\prime}} .
$$

Taking into account the negative value of $\psi^{\prime}$ we have $\psi^{\prime}=-\left|\psi^{\prime}\right|$. In this case the Equation (12) can be written as

$$
l=\varphi(T)=\frac{\psi_{0}+1}{\left|\psi^{\prime}\right|}-\frac{2 T\left[\psi_{0}+1-C_{1}\left|\psi^{\prime}\right|\right]}{T_{\max }\left|\psi^{\prime}\right|} .
$$

Thus, upon known values of $\psi_{0},\left|\psi^{\prime}\right|, T_{\max }$, assuming existence of linear scale of values of $T$, we can calculate $l$ using formula (13).

Then, using the composed set of values $\left\{T_{i}\right\}, i=\overline{1,3}$ where elements of this set increase linearly on index $i$, the elements of the set $\left\{l_{i}\right\}, i=\overline{1,3}$ should be calculated. Using the calculated values of $l_{i}, i=\overline{1,3}$, the geometrical construction of highly informative zone, which may or may not contain the MR, should be carried out.

\section{Conclusions}

Thus, the new notion, maximum informative zone, suggested in this work, makes it possible to remove the initial vagueness concerning selection of minimum network of RS for determining of error of mobile receiver's position using the interpolation method.

It should be noted that in this case the two critical conceptions for determining of error of MR-s position (using criteria of maximum interpolation accuracy and maximum amount of information) don't follow up the classic concept of multicriterial optimization, because the competition of criteria is absent here and requirement to meet one of them could mean fulfillment of other one.

\section{REFERENCES}

[1] H. Vollath, U. Vollath and X. Chen, "Virtual Reference Station Systems", Journal of Global Positioning Landau Systems, Vol. 1, No. 2, 2002, pp. 137-143. http://dx.doi.org/10.5081/jgps.1.2.137

[2] U. Vollath, H. Landau, X. Chen, K. Doucet and Ch. Pagels, "Network RTK versus Single Base RTK Understanding the Error Characteristics,” ION GPS2002, Portland, 24-27 September 2002, pp. 2774-2781.

[3] U. Vollath, A. Deking, H. Landau and Ch. Pagels, "LongRange RTK Positioning Using Virtual Reference Stations," International Symposium on Kinematic Systems in Geodesy, Geomatics and Navigation, Banff, June 2001, pp. 470-474.

[4] Y. Feng and J. Wang, "GPS RTK Performance Characteristics and Analysis,” Journal of Global Positioning Systems, Vol. 7, No. 1, 2008, pp. 137-143. http://dx.doi.org/10.5081/jgps.7.1.1

[5] G. Badesku, O. Stefan, R. Badesku, M. Ortelecan and S. I. Veres, "Positioning System GPS and RTK VRS Type, Using the Internet as a Base a Network of Multiple Stations," FIG Working Week 2011, Bridging the Gap between Cultures Marrakesh, Morocco, 18-22 May 2011, pp. 1-11.

[6] F. Takac and O. Zelzer, "The Relationship between Network RTK Solutions MAC, VRS, PRS, FKP and i-MAX,” Proceedings of ION GNSS, Savannah, 16-19 September 2008, pp. 2379- 2387.

[7] L. Wanninger, "The Performance of Virtual Reference Stations in Active Geodetic GPS-Networks under Solar Maximum Conditions," Proceedings of ION GPS, Nashville, 16 September 1999, pp. 1419-1427.

[8] H. Landau, U. Vollath and X. Chen, "Virtual Reference Stations versus Broadcast Solutions in Network RTKAdvantages and Limitations,” GNSS, Graz, 2003, pp. 115.

http://gootechmabna.com/catalouges/Papers/3sencore/15. pdf

[9] S. Wu, K. Zhang and D. Silcock, "Magnitudes and Temporal Variations of the Tropospheric and Ionospheric Errors in GPSnet," Journal of Global Positioning Systems, Vol. 9, No. 1, 2010, pp. 61-67. http://dx.doi.org/10.5081/jgps.9.1.61

[10] S. Wu, K. Zhang, Y. Yuan and F. Wu, "Spatio-Temporal Characteristics of the Ionospheric TEC Variation for GPS Net-Based Real-Time Positioning in Victoria," Journal of Global Positioning Systems, Vol. 5, No. 1-2, 2006, pp. 52-57. http://dx.doi.org/10.5081/jgps.5.1.52 